\title{
LA TEORIA DE LA AROMATICIDAD: UN PROGRAMA DE INVESTIGACION
}

Fuentes Medina, G. P y Muñoz Albarracin, L. M. gpilarfuentes@hotmail.com ; lizmayoly@hotmail.com

Estudiantes, Maestría en Docencia de la Química. Universidad Pedagógica Nacional.

\section{RESUMEN}

En el presente artículo se discurre sobre la construcción de la teoría de la aromaticidad partiendo de la propuesta de August Friederich Kekulé en 1865; se tienen en cuenta los aportes hechos a la misma por otros autores, los cuales fundamentaron la teoría dando explicación a los interrogantes que surgieron durante su elaboración para ser aceptada por una comunidad científica .

El análisis que se pretende realizar, parte de la reconstrucción de la historia interna, desde una aproximación a la propuesta Lakatosiana, en la que se examinan los problemas que la teoría resolvió, las preguntas que no respondió, los programas de investigación en competencia y finalmente los aportes a la comunidad científica.

PALABRAS CLAVES: Aromaticidad, atomicidad, Tetravalencia, Historia interna, teoría de tipos, teoría de radicales, teoría de sustitución, teoría electrónica de valencia, regla de Hückel.

\begin{abstract}
In this article we want to discuss about building aromaticity theory since August Friederich Kekule's proposal made it in 1865; we must release the contributions make to its by others authors no less importants, which given a solid bases to the theory and a satisfactory explications to several questions that emerged during the time its was built for be to accepted by a scientifican community.
\end{abstract}

The analysis that we wish to do, begin with the internal history reconstruction since an approach Lakatos's works, for what we will review the problems that the theory can solve, the questions that it can't give an answer, the investigations programs which was in competence each other and finally the contributions to the scientifican community .

KEY WORDS: Aromaticity, atomicity, tetravalency, internal history, types theory, radical theory, sustitution theory, electronic theory of valence, Hückel's rule.

\section{INTRODUCCIÓN}

Incluir el desarrollo histórico y epistemológico de las teorías o modelos científicos, significa acceder a los marcos conceptuales y metodológicos que explican como ha sido el proceso de construcción de las mismas, lo cual incide en el mejoramiento de los procesos de enseñanza.

Como consecuencia es necesario elaborar una historia de las ciencias, que permita mostrar que el conocimiento se construye a partir de un sin número de formulaciones, experiencias puestas en tela de juicio por la sociedad, de modo que quede claro que las ciencias no se producen a partir de verdades absolutas e irrefutables; al mismo tiempo que la historia no es una narración de hechos puntuales, como tampoco una colección de descubrimientos. 
Así, bajo el marco de la historia de las ciencias experimentales, el componente epistemológico permite conocer el mecanismo de producción de los conocimientos, mediante el uso de estrategias didácticas que tengan en cuenta las formas de aprendizaje del estudiante y desarrollen habilidades de pensamiento para la utilización de estos conocimientos científicos. (Gagliardi y Giordan, 1986).

Como se mostrará, la teoría de la aromaticidad tiene sus fundamentos conceptuales en la teoría de los radicales (Berzelius, 1837), la teoría de sustitución (Dumas, 1848) y la teoría de los tipos (Gerhardt,1849); recurriendo al desarrollo histórico de estas teorías, encontramos que por ese entonces, la mayoría de los químicos se encontraba proponiendo nuevos procedimientos para la síntesis de compuestos orgánicos, que permitieran corroborar la existencia de dichas teorías. En este intento, las profundas investigaciones de Frankland (1852),sobre los compuestos orgánicos de nitrógeno, fósforo, arsénico y antimonio, permitirían aclarar el comportamiento de los compuestos orgánicos basados en que todo elemento debía tener una valencia determinada, o, capacidad de saturación que, sin embargo podía presentarse con diferentes magnitudes. Con lo anterior se puede dilucidar como Kekulé basado en dichas conclusiones determinaría la tetravalencia del carbono.

De este modo, los trabajos realizados por Kekulé (1865) sobre la tetravalencia del carbono y la conformación de cadenas carbonadas en los cuerpos grasos (hidrocarburos alifáticos), lo conducirían a formular su teoría de los compuestos aromáticos. Así, en cuanto a la trascendencia de la teoría propuesta por Kekulé, se analizará por qué se asume como programa de investigación científica, de que maneras fue un programa progresivo y de la misma forma, que campo delimitó, qué preguntas respondió, cuáles las anomalías que presentó, si hubo o no programas en competencia y en que consistieron dichos programas..

\section{HISTORIA INTERNA}

El análisis que aquí se aboca, busca hacer una reconstrucción racional desde la historia interna de la teoría de la aromaticidad. Se ha tomado a Lakatos (1983) como referencia para explicar la estructura lógica de la teoría de Kekulé; se intentará realizar la descripción de los hechos que contribuyeron significativamente a la formulación de esta teoría. Por historia interna, explica Lakatos, ha de entenderse la "reconstrucción racional del crecimiento objetivo" de un programa, lo que depende de la aproximación filosófica adoptada por quién se ocupa de esta problemática

La Química orgánica no tuvo sus inicios siguiendo un plan específico. Fueron algunos experimentos casuales con compuestos inorgánicos los que condujeron al reconocimiento de sustancias diferentes a las que se venían trabajando. El origen de esta química se da entonces con el estudio de los compuestos que se obtenían de los animales y vegetales, los cuales estaban constituidos por carbono e hidrógeno (Lavosier, 1789); además con la síntesis de la urea obtenida a partir de cianato amònico por Wohler en 1828 y a partir de sales de ácido fulmìnico obtenida por Liebig en el mismo año, empieza a darse a conocer la síntesis de los compuestos orgánicos.

Para las nuevas sustancias sintetizadas, Berzelius (1840) empezó a elaborar un orden sistemático, a partir del cual planteó la teoría de los radicales, basado en que, en las síntesis realizadas existía un grupo de átomos de características semejantes, que abundaban en los compuestos orgánicos. A estas series de átomos las llamo "radicales", que según él, no variaban en su composición. Aunque los documentos originales indican que Liebig y wöhler 
(1832) son los primeros en aportar datos a la Teoría de los Radicales con el radical benzoilo, finalmente es Berzelius quien publica los resultados de esta investigación.

A los grupos de átomos que Berzelius decía que no variaban esencialmente en su carácter por la sustitución del hidrógeno, Dumas los llamó “tipos", pero es Laurent quien propone La Primera Teoría de Tipos (1836). Posteriormente Jean Baptiste Dumas demostró que en estas series se podían sustituir el hidrógeno por el cloro, proponiendo la Teoría de Sustitución(1840), la cual contribuyó a esclarecer el concepto de equivalente. Más tarde Charles Gerhardt (1849), amigo y colaborador de Laurent, denominó "residuos" a los componentes que en las reacciones de los compuestos químicos se unen unos con otros con eliminación de átomos; además afirmó que tales residuos no existían en estado libre. Con esto se establece La Segunda Teoría de Tipos(1849), la cual fue producto de una combinación de La Teoría de los Radicales(1838) con la Teoría de Sustitución (1840).

La nueva Teoría de Tipos consistía en definir estructuras fundamentales "Tipos", a partir de los cuales podían obtenerse las formulas de numerosos compuestos mediante la sustitución de átomos de hidrógeno por radicales más o menos complejos. Gerhardt distinguió cuatro "tipos" inorgánicos: agua, amoniaco, ácido clorhídrico e hidrógeno, a partir de los cuales podían derivarse, en principio todos los compuestos orgánicos. Así "el metanol $\mathrm{CH}_{3} \mathrm{OH}$, pertenecería al tipo del agua, habiéndose sustituido uno de los hidrógenos del $\mathrm{H}_{2} \mathrm{O}$ por el radical $\mathrm{CH}_{3}$ " ( Garrat, 1974).

Mientras se discutía cuales eran las teorías que explicaban el comportamiento de la mayoría de los compuestos orgánicos encontrados se perdía de vista la importancia del concepto de átomo, el cual daría las bases para la química orgánica estructural. Archibald Couper (1858) se interesó en proponer, mediante modelos espaciales, una explicación al concepto de "atomicidad" como unidad de afinidad que se establece entre los átomos, estas fuerzas que unían los átomos las representó por líneas punteadas que proyectarían los enlaces entre estos. Crum Brown`s (1860) refina las líneas de representación de Couper aportando también al campo de la química estructural.

Al mismo tiempo que Couper realizaba sus trabajos Kekulé propone la Teoría de la Tetravalencia del Carbono (1858) y la posibilidad que tiene este elemento de enlazarse formando cadenas sin ahondar en la estructura de las mismas. Los avances en la representación de las moléculas continuaron con los trabajos de Lewis (1916) quien propuso representar los electrones de valencia que se involucran en el enlace dando lugar junto con los aportes posteriores de Hückel (1931) a la estructura electrónica del benceno la cual define la regla para considerar a un compuesto como aromático o no.

Con el desarrollo de estas teorías se definieron conceptos básicos, tales como átomo, molécula, equivalente y la determinación de pesos moleculares que fueron aceptados por la comunidad científica, por otro lado conceptos poco delimitados como valencia, en ese entonces denominado "atomicidad"(Kekulé, 1865) ó unidades de afinidad hacían que fuera difícil llegar aun consenso y por tanto no se contaba con una estructuración teórica con la cual organizar los resultados experimentales obtenidos.

La reconstrucción racional de la historia interna nos permite identificar cuales han sido los aportes hechos en primera instancia a la conformación de la química orgánica estructural 
permitiéndole a kekulé, sentar las bases de la química que le daría los fundamentos conceptuales para explicar su teoría de la aromaticidad, para ello recurre a establecer las propiedades químicas estructurales entre los compuestos alifáticos y los aromáticos.

\section{CONSTRUCCIÓN DE LA TEORIA DE LA AROMATICIDAD}

Los estudios que condujeron a la postulación de la teoría de la aromaticidad se pueden analizar, como se dijo, siguiendo la metodología propuesta por Lakatos (1983), así para los responsables del presente artículo, el núcleo firme de este programa de investigación estuvo conformado por los siguientes postulados (Kekulé 1865):

1. La tetravalencia del carbono en los compuestos aromáticos y en los cuerpos grasos.(compuestos alifáticos) Kekulé (1865)

2. El convencimiento de que todos los compuestos aromáticos, aun el más simple son proporcionalmente más ricos en carbono que los compuestos homólogos en la clase de los cuerpos grasos.

3. En los compuestos aromáticos, al igual que en los cuerpos grasos, hay numerosas sustancias homologas, como aquellas en las que las diferencias de composición pueden ser expresadas por la formula $\mathrm{nCH}_{2}$, en donde $\mathrm{n}$ es un número entero.

4. El más simple compuesto aromático contiene por lo menos seis átomos de carbono

5. Todas las alteraciones de las sustancias aromáticas muestran ciertamente una similaridad familiar por tanto estos pertenecen al grupo de los "compuestos aromáticos".En las reacciones de sustitución, una parte del carbono es frecuentemente eliminado, pero el producto principal contiene por lo menos seis átomos de este elemento.

6. En las sustancias aromáticas se supone la existencia de núcleos comunes; esto es, una cadena cerrada $\mathrm{C}_{6} \mathrm{~A}_{6}$ (donde $A$ significa una unidad insaturada o una unidad de afinidad).

Los anteriores postulados fueron protegidos por las siguientes hipótesis auxiliares, que hicieron las veces de cinturón protector (Kekulé 1865):

1. En toda sustancia aromática hay contenidos de uno o de varios grupos de seis átomos de carbono, ó si se desea un núcleo que consiste de seis átomos de carbono.

2. En estos núcleos los átomos de carbono tienen una combinación cerrada o una organización más compacta.

3. A estos núcleos, más átomos de carbono pueden ser adicionados como en el caso de los cuerpos grasos. Sí muchos de los átomos de carbono pueden ser unidades por si mismas, entonces esto también puede suceder con estos núcleos, ya que una unidad de afinidad de un átomo puede unirse con otra unidad de afinidad de un átomo vecino. Se asume, de este modo puesto que es la forma como dos átomos de carbono permanecen unidos.

Las dos últimas hipótesis pueden ser representadas por las siguientes reacciones de simetría $1 / 1,1 / 1,1 / 1,1 / 1$, etc; $1 / 1,2 / 2,1 / 1,2 / 2$, etc. La primera de estas representaciones corresponde a los cuerpos grasos y la segunda da una explicación de la constitución de las sustancias aromáticas. 
4. Sí se acepta que los seis átomos de carbono se unen de acuerdo a relaciones de simetría, se obtiene un grupo que es formulado por una cadena abierta, que contiene ocho unidades afines insaturadas. Sí se supone además que los dos átomos de carbono finales de la cadena se unen por las unidades afines, entonces se obtiene una cadena cerrada (un anillo simétrico) el cual ya contiene seis unidades afines libres.

5. Las seis unidades afines de estos núcleos pueden ser saturados por seis elementos monoatómicos 0 , por, una parte de ellas, pueden ser saturadas por unidades de elementos poli atómicos afines pero estos más tarde deberán ser unidos a otros átomos, para formar una o más cadenas laterales, las cuales pueden ser alargadas ampliamente, por uniones entre ellas mismas o con otros elementos.

6. Una saturación de dos unidades afines del núcleo considerado por un átomo de un elemento diatómico o una saturación de tres unidades afines por átomos de elementos triatómicos no es posible, en teoría. Los compuestos de formula molecular $\mathrm{C}_{6} \mathrm{H}_{4} \mathrm{O}$, $\mathrm{C}_{6} \mathrm{H}_{3} \mathrm{~N}, \mathrm{C}_{6} \mathrm{H}_{4} \mathrm{~S}$ son impensables.

Los postulados anteriores han constituido la base teórica que definen y explican el comportamiento, características, propiedades físicas y químicas de los compuestos orgánicos alifáticos y aromáticos, desde el programa de investigación lakatosiano el núcleo firme desde su consolidación ha permanecido vigente gracias al apoyo conceptual que las hipótesis auxiliares le han proporcionado al programa.

\section{APOYOS EMPIRICOS DEL PROGRAMA DE KEKULE}

La teoría de la aromaticidad se sustenta bajo los siguientes trabajos experimentales:

1. La síntesis del xilol realizada por Rudolph Fittig y Bernhard Tollens por acción del sodio sobre una mezcla de bromobenzol y yoduro de metilo y la síntesis del etilbenzol a partir del bromobenzol y yoduro de etilo como producto inesperado. Esta síntesis y sus resultados condujeron a Kekulé a deducir la formula del benzol, como un compuesto en los que se unen los átomos de carbono por enlaces sencillos y dobles alternos, formando un anillo.

2. La síntesis del benceno a partir del etino: tres moléculas de etino se condensan en una de benceno, por acción del calor; lo cual se explica suponiendo que una valencia de los etino se abre para servir de enlace.

3. La síntesis de homólogos del benceno, partiendo de las cetonas, con un volumen igual de ácido sulfúrico diluido $1 / 1$, dejándolo en reposo un día entero y destilando. Se obtiene el 1,3,5 trimetilbenceno y análogamente el 1,3,5 trietilbenceno; productos que contienen tres dobles valencias alternadas con otras tres sencillas.

4. La obtención de derivados monosustituidos, cuyas formulas son siempre superpuestos mediante rotación.

5. La presencia de tres isómeros distintos llamados orto, meta y para.

6. Las dificultades que el derivado 1,6 presenta puesto que predice que no es superpuesto sobre el 1,2,ya que éste último posee un enlace simple entre los dos carbonos, mientras que el 1,6 lo tiene doble. Este hecho supone un cuarto isómero disustituido que no se ha encontrado y que en realidad no existe.

Para explicar esta anomalía Kekulé tuvo que expandir su cinturón protector proponiendo que el doble enlace, no es de fijeza absoluta, sino que es de naturaleza oscilatoria, en un 
momento está entre $C_{1}$ y $C_{2}$ y en el otro entre $C_{1}$ y $C_{6}$. Esta propuesta que no fue aceptada fácilmente, puesto que conducía a pensar en una inestabilidad en el anillo, que debería aplicarse a las otras dos dobles valencias, de modo que esa valencia móvil, debería quedar libre en el interior de la estructura. Para admitir tal valencia móvil, se requirió de otras hipótesis adicionales sugeridas por otros científicos, las que se explicarán más adelante.

7. Los resultados de las pruebas de refractometría realizados confirman el peso molecular determinado por otros procedimientos.

8. La formación de un triple ozónido, característica también de los compuestos eténicos. El triple ozónido al descomponerse con el agua da tres moléculas de etanodiol y tres moléculas de agua oxigenada.

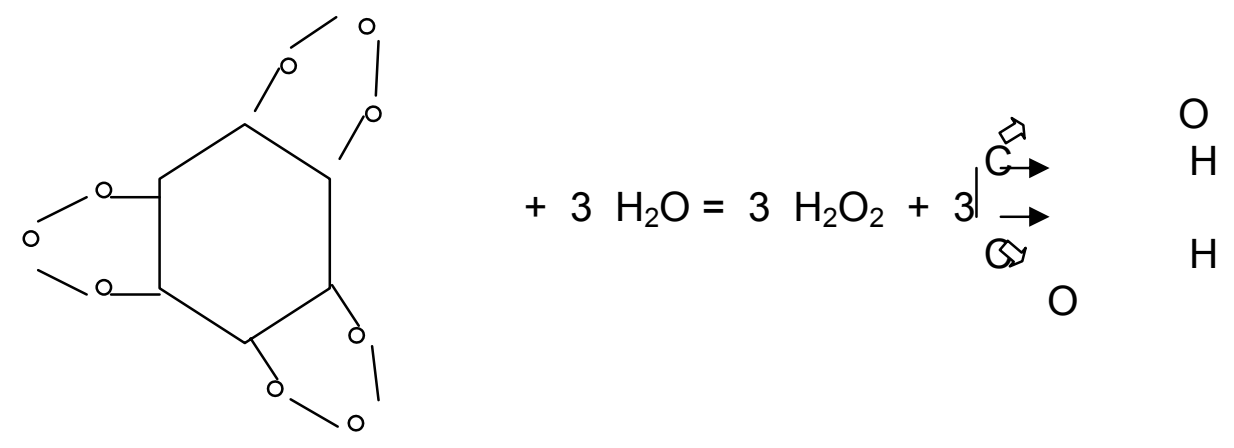

\section{TRIPLE OZONIDO}

\section{PROPUESTAS ALTERNATIVAS}

La construcción de la teoría de la aromaticidad propuesta por Kekulé tuvo que superar las propuestas de Claus y Koerner, Armstrong y Baeyer, Landerburg, Thiele y Dewar. Partiremos de su estudio estructural, junto con las razones que las sustentan y las que no las validaron, determinando de este modo que le permitió a Kekulé afirmar su teoría y al mismo tiempo concluir porque estas propuestas constituyen una progresión y consolidación de la teoría de la aromaticidad como un programa de investigación.

\section{ESTRUCTURA DE CLAUS Y KOERNER (1867)}<smiles>CC12C3C4C5C3C1C5(C)C42</smiles> 
Las razones que apoyan la formulación de esta estructura supone que las cuatro valencias de cada carbono se satisfacen con el carbono opuesto; por esto se le llamó diagonal. Con ella es explicable la imposibilidad del cuarto isómero disustituido al cual se aludió la ausencia de las reacciones eténicas

Las razones que contradicen en general son las que apoyan la teoría de Kekulé, además admite isómeros ópticos desde el momento en que cada carbono está unido a un cuarto carbono, que pueden convertirse en radicales distintos quedando este carbono asimétrico, sin embargo no se conocen derivados del benceno óptimamente activos. La formula muestra una saturación, por tanto, no explica la formación de derivados de adición (6 bromos, 6 cloros, 6 hidrógenos). Además la experiencia dice que la entrada de $\mathrm{Br}_{2}$,por ejemplo, se hace siempre en posiciones inmediatas y no diagonales. No es clara la diferencia entre el isómero orto y para: pues ambos enlaces son directos. Posee implicaciones estereoquímicas al considerarlas como estructuras tridimensionales.(Vitoria 1940)

\section{ESTRUCTURA DE ARMSTRONG (1887) Y BAEYER (1888)}

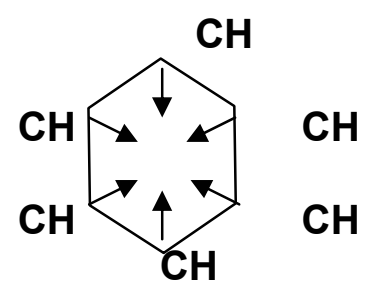

Una de las razones que apoyan esta formula, supone que las cuatro valencias de los seis carbonos, se unen en el centro del hexano, saturándose los seis al mismo tiempo como si formasen un nudo. Por eso se llama céntrica. También explica porque el benceno no da derivados de adición como los compuestos eténicos. Igualmente, la resistencia a la ruptura que ofrece este anillo, ya que son seis las valencias que mutuamente se saturan en el centro. Da cuenta de porque, si, por la razón que sea, se rompe el anillo, el sexto enlace, ya presenta en este anillo los caracteres de grupo eténico, pues la solidez de la formula está en que las seis valencias se saturan en el centro. En el momento en que alguna ceda ya no hay razón alguna de ese equilibrio.

Explica la falta de reacción con el etanoato mercúrico y el reactivo de Baeyer además con ella tampoco es posible el cuarto isómero.

Entre las razones que la contradicen, está la síntesis a partir del etino en la que no se explica tan naturalmente, la existencia de triozónidos, la refractomería, la unión de las seis cuartas valencias en el centro es nueva, indirecta y compleja, cuya necesidad no se ve justificada.. No explica la falta de isómeros ópticos ya que según la representación estereoquímica, deben poder existir tales derivados activos, mientras que la propuesta por Kekulé no los admite. Finalmente con esta estructura, no se explican los enlaces mutuos de varios anillos constituyendo núcleos más complejos, como antraceno, fenantreno. Años más adelante el naftaleno y el pirrol se consideran excepciones a la estructura de Baeyer (Garratt 1974) y 
demás, aunque parece que Willstaetter confirmó una análoga constitución céntrica en el cliclooctatetreno, esto no indica que se considere un compuesto aromático (Vitoria, 1940)

\section{ESTRUCTURA DE LADENBURG (1874)}

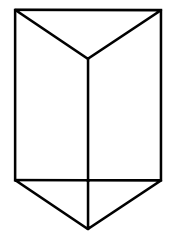

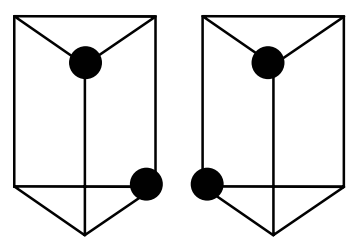

Fig. A
Fig. B

Las razones que la apoyan son todas las que justifican la falta de enlaces eténicos. Es consistente con el hecho de la existencia de un benceno disustituido.

Las razones que la contradicen, son las que favorecen la formula de Kekulé. Es inevitable la posibilidad de isómeros ópticos: porque aunque se prescinde de la representación estereoquímica del átomo de carbono, ésta formula siempre es espacial, por tanto, los carbonos asimétricos aquí son posibles en infinidad de casos, de modo que implica la existencia de formas "orto"enantioméricas. Con esta no se evita el cuarto isómero como lo indican las figuras A y B que son superpuestas( Vitoria , 1940).

\section{ESTRUCTURA DE THIELE (1899)}

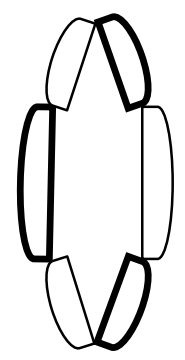

Esta formula está fundada en la teoría de los dobles enlaces conjugados de Kekulé. Según esta, como cada doble enlace se alterna con otro simple, resulta que las valencias residuales se unirán también de dos en dos y quedaran los enlaces arqueados alternativamente recios y punteados. Si se supusiera que la valencia segunda de la doble unión se fraccionara en dos partes iguales, la formula del benceno según Thiele, se presentaría como un hexágono regular, los carbonos de cuyos vértices estarían unidos entre sí, todos, por enlace lineal y enlace curvilínea, de igual grosor

Entre las razones que la apoyan está la desaparición del enlace eténico quedando todos inactivos y por tanto no hay para que exigir del benceno las reacciones características de esta doble unión tantas veces dicha. Sí se supone que las valencias residuales son iguales que las principales, desaparece el cuarto isómero de Kekulé. Thiele siempre pensó que la valencia residual era distinta y menor que la principal: de ser así, es un error suponerlas iguales y, si no son iguales, ya no desaparece el cuarto isómero (Vitoria, 1940).

La estructura no se puede justificar ya que los fundamentos que presenta no son admisibles. La refractomería, es una prueba de esto, al igual que la formación de triozonidos. Según 
esta propuesta la formula del benceno queda reducida a la de un compuesto saturado. La existencia del ciclooctatetraeno invalida las suposiciones de Thiele al presentar características de oleofina y no de aromático.

\section{ESTRUCTURA DE DEWAR (1945)}

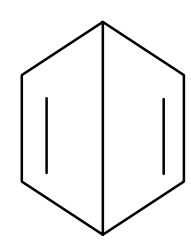

La única diferencia de la estructura de Dewar es plantear otro tipo de oscilación para los dobles enlaces, y no presenta otro tipo de ventajas o desventajas comparada con la de Kekulé, razón por la cual no es muy conocida y apenas se menciona en los textos.

De acuerdo con el programa de investigación mostrado es posible hacer algunas reflexiones en torno a si permitió avanzar en el campo de la química orgánica o, por el contrario, no aportó mayores significaciones ( Vitoria, 1940).

\section{PROGRESIÓN DEL PROGRAMA DE INVESTIGACIÓN}

A pesar de las anomalías que se presentaron con la estructura propuesta por Kekulé, estas dificultades no impidieron que el programa avanzara, por el contrario, todos los hechos que se plantearon como restricciones a la teoría, algunos años después sirvieron para corroborar la eficacia de las hipótesis formuladas.

Las demás estructuras propuestas no tuvieron la trascendencia necesaria como para sustituir o modificar la teoría de Kekulé. El éxito de este programa de investigación radica precisamente en la solidez de sus postulados, la capacidad para predecir hechos nuevos, de corroborar los hechos vigentes y los que le dieron origen. También se destaca por la ingeniosa habilidad y creatividad requeridas para salvar el núcleo firme, con la introducción de modificaciones a las hipótesis que conforman el cinturón protector.

El poder explicativo de la teoría impidió que se formularan otros programas de investigación en competencia. El progreso de dicho programa, es tal que los descubrimientos posteriores como por ejemplo el del electrón corroboraron mucho más la eficiencia de la teoría mejorándola y extendiéndola, sin que se modificara el núcleo firme que hasta el día de hoy se mantiene y ha sido la base para construir una rama de la química como lo es la química estructural, la que permitió el desarrollo vertiginoso de la química orgánica .

\section{¿QUÉ CAMPO DELIMITA?}


Kekulé pretendió en un comienzo dar respuesta teórica a los resultados obtenidos en la síntesis de compuestos alifáticos, los que en ese momento carecían de explicación científica; posteriormente, establece la diferencia entre compuestos aromáticos y alifáticos describiendo, detenidamente las reacciones típicas de algunos de estos grupos especialmente las de los aromáticos. Kekulé establece los parámetros para dar nacimiento a la Química estructural. Esta disciplina fue acrecentada por los trabajos de Beayer, Ingol, Huekel, Robinson, Markovnikov, Pauiling , Lewis Wurtz entre otros.

Del articulo se puede inferir que el estudio de la Teoria de la Aromaticidad tiene como antecedentes los trabajos realizados desde Lavoisier (1789), Liebig y Wohler(1832), Dumas (1840) Gerharth (1849) y otros; en cuanto a las investigaciones posteriores encontramos las llevadas a cabo por Lewis (1916), Paulling (1926) y Hückel (1931).

\section{PROBLEMAS QUE RESUELVE}

El programa de investigación de Kekulé da respuesta a las distintas formas de síntesis del benceno, hechas hasta ese momento; justifica la aparición de los tres isómeros disustituidos; la presencia de un solo compuesto monosustituido; predice la pobreza de las reacciones de compuestos derivados del benceno; y, da razón de la existencia de compuestos aromáticos formados por varios anillos.

En conclusión el aporte más importante es que da respuesta a formulación de una estructura bencénica que satisface y da explicación a la mayoría de las propiedades químicas y físicas que presenta el benceno; sin dejar de lado que los aportes posteriores brindan mejores explicaciones y resuelven algunas dificultades, sobre todo en cuanto a su estructura electrónica se refiere.

\section{¿QUÉ NO RESPONDE?}

No es posible indagar acerca de la divalencia del carbono; no justifica el porque no se obtienen derivados de adición con los halógenos, en frío y con facilidad, algo que es propio de enlaces eténicos; no da cuenta de la falta de reactividad con el acetato mercúrico en solución saturada y fría, reacción usual del doble enlace eténico; con esta estructura no hay reacción con el reactivo de Baeyer propio también de todos los enlaces eténicos; y no justifica el cuarto isómero disustituido posible con esa estructura.

\section{APORTES DE LA COMUNIDAD DE ESPECIALISTA A LA TEORIA DE KEKULÉ}

Kekulé, trabajaba en la universidad de Heidelberg, y por ese tiempo se enteró de las investigaciones realizadas acerca de las suposiciones de la valencia o la "atomicidad" del carbono, empezó a discutir y organizar toda esta información. Años más tarde, Kekulé gracias a sus habilidades de proyección espacial y a su capacidad de síntesis pudo exponer una teoría más clara del problema en 1858.

Pero no fue el único en proponer la teoría estructural, ya que Archibald Scott Couper (1858), independientemente se encontraba, diseñando una forma de representación del ácido tartárico y del producto que se obtenía de la deshidratación de éste por acción del calor. En 
su publicación propone el uso de líneas continuas y corchetes para representar los enlaces. Estas líneas le sirvieron a Kekulé para representar las formulas de constitución de sus compuestos aromáticos, una de las razones por las cuales se considera el fundador de la química estructural.

En efecto, Couper consideró los siguientes puntos como parte de su propuesta: Considerar la química como una sola y completa; tomar en consideración cada combinación conocida y estudiar el carácter las funciones y propiedades mostradas para cada elemento en cada una de sus combinaciones junto con las diferentes condiciones y aspectos, esto con el fin de comparar los diferentes cuerpos entre sí, ya que se dispone de cada parte de un todo que es realizado por cada elemento separadamente; y, proponer los principios generales comunes a todos los elementos así como las propiedades especiales de cada uno.

Este método lo aplicó para presentar consideraciones particulares del elemento del carbono las cuales tienen dos grandes características: El carbono se combina con igual número de hidrógenos, cloros, oxígenos, azufres y puede enlazarse químicamente consigo mismo, propiedades estas que, según Couper (1858), explican todas las características de la química orgánica .

La química estructural tuvo sus primeras investigaciones a raíz del artículo publicado por Kekulé (1865)en el que intentó aclarar el problema de la validez de la valencia del carbono, con base en las combinaciones saturadas llamadas oleofinas, puesto que al contrario que en las parafinas, el carbono no enlaza sus cuatro valencias con otros átomos.

Loschmith (1860) introdujo el concepto del doble enlace para dar explicación a ello, así como hizo las primeras representaciones de las posiciones relativas de los átomos en el espacio y la expresión gráfica de ciertos fenómenos de isomería. Hoffman ( 1865), introduce más tarde las terminaciones ano, eno, e ino para caracterizar los hidrocarburos saturados e insaturados.

Los trabajos realizados por Fitting y Tollens acerca de la síntesis del xilol proporcionaron a Kekulé (1865) la base para dirigir sus investigaciones hacia la concepción cíclica y de enlaces sencillos y dobles alternados para el compuesto bencénico.

A continuación se muestran algunas de los diagramas con los que Kekulé ( 1865) empezó a representar al benceno. Con estos trazos el no quiere denotar el arreglo espacial de los átomos. Para su segundo documento, ya propone el hexágono. En las figuras, las elipses representan los átomos de carbono; las líneas los enlaces entre los átomos de carbono; los puntos las zonas de unión de los átomos de Hidrógeno y las flechas indican la unión de los átomos de Carbonos terminales. (Garrat, 1974).

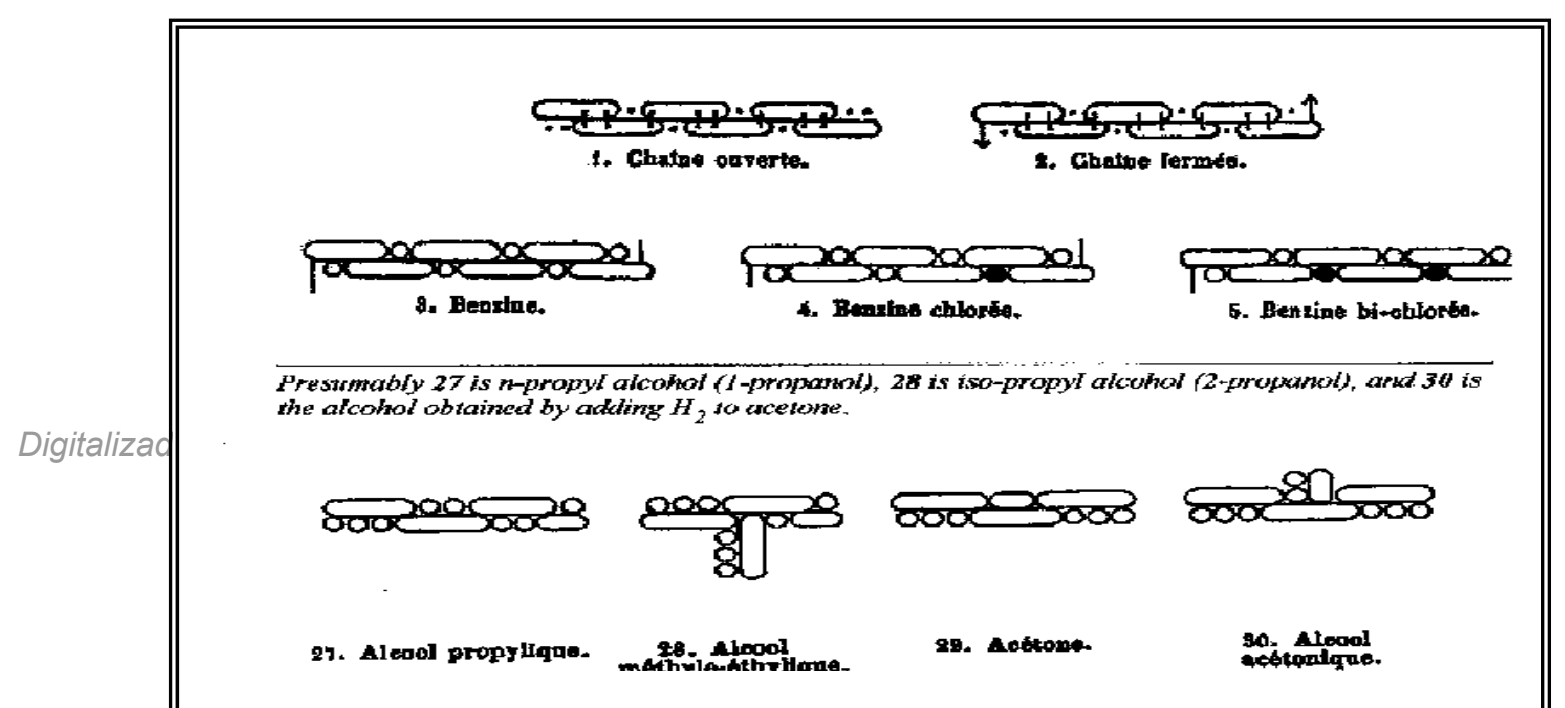


Ésta primera hipótesis debió variar un poco puesto que no era coherente frente a ciertos resultados experimentales encontrados, como por ejemplo, la ausencia de isómeros orto del benzol; por esta razón Kekulé (Dewar 1866) propone un estado oscilatorio de los enlaces de carbono.

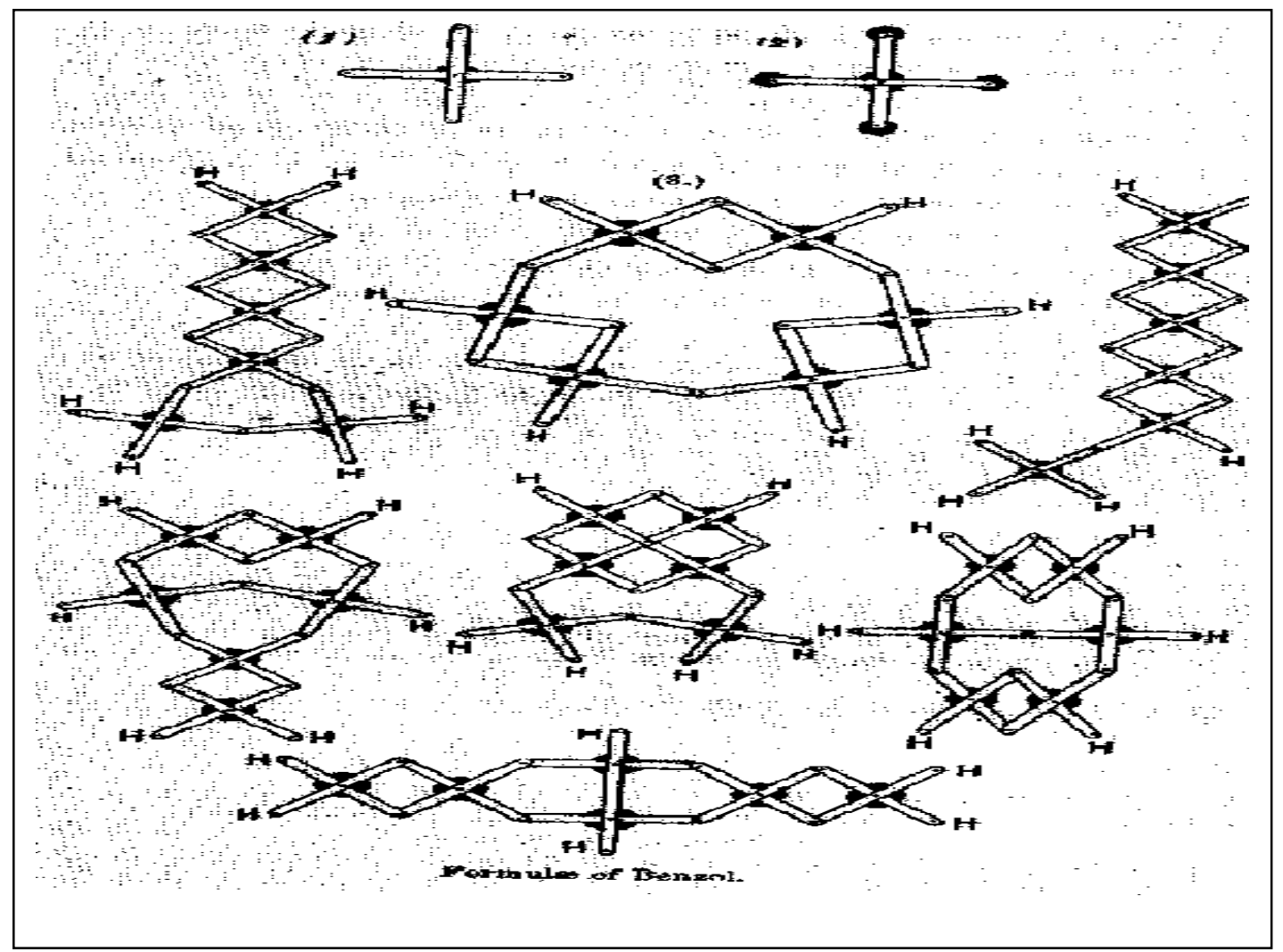

Con los diagramas mostrados, se puede entender la forma como Kekulé finalmente le dio forma a la propuesta de la estructura del Benceno, ayudado por las ideas de Crum Brown, Dewar y otros.

A pesar de hacer varias propuestas estructurales para el benceno, tampoco Kekulé fue el único en llegar a la misma conclusión, ya que Loschmith en 1861 propone para el benzol un núcleo del carbono hexatómico, que consideraba como un elemento hexavalente y lo representó gráficamente por un circulo con seis puntos de enlace, pero no pudo explicar la isomería de los tres cresoles; motivo por el que fue ignorado.

Aún así la estructura de Kekulé no fue aceptada tan fácilmente debido a la presencia de algunos productos de reacciones imprevistos desde la teoría. Es entonces cuando se dan a conocer las estructuras formuladas por: Claus, Koerner, Landerburg, Deawer, meyer, Armsntrong; las cuales no tuvieron la gran amplitud explicativa de la propuesta por Kekulé, 
de modo que, al no presentarse predicciones importantes a partir de estas estructuras, fueron perdiendo validez los postulados en los que se apoyaban consolidándose la idea del anillo aromático de Kekulé.

Las posteriores investigaciones junto con los recientes descubrimientos permitieron corroborar la imposibilidad de reacción típica de los compuestos aromáticos (reacciones de adición, entre las que se encuentran la formación de polímeros naturales de los alquenos), explicado por la teoría electrónica y posteriormente por la teoría de la hibridación.

Los avances más importantes para la fundamentación teórica de la química estructural vendrá después con la admisión de la existencia del electrón y el auge de las discusiones referidas a la estructura atómica.

Todos ellos corroboran las primarias concepciones de Kekulé, como el acontecimiento más importante en la química orgánica de ese entonces. La teoría electrónica sustituyó a la teoría de valencia, para la explicación del comportamiento de los enlaces en el anillo de benceno.

Un aporte necesario a la propuesta que aquí se hace acerca del trabajo de Kekulé y otros para consolidar la teoría de la aromaticidad como un programa de investigación, desde la perspectiva Lakatosiana, podría venir de la aplicación del método de los orbitales moleculares de Hückel (Garrat, 1974) a partir de lo cual " los sistemas aromáticos son moléculas monocarbocíclicas conjugados que contienen $(4 n+2)$ electrones $\pi$, fuera del plano".

Pero a pesar de incluir al electrón dentro de la definición y el despliegue derivado de la cuántica es una definición que presenta limitantes para designar a todos los compuestos aromáticos. Aunque se cuenta con aproximaciones como la de la teoría molecular de Huckel cualquier definición no resulta completamente satisfactoria, si las consideraciones para tal propósito están basadas en su estructura o en el estado fundamental o en el estado de transición propio de las reacciones o de la diferencia energética entre los estados anteriores.

Muy probablemente el intento para clasificar a un compuesto como aromático o no pueda estar basado en la combinación de la teoría de los orbitales moleculares, la teoría de Hibridación y la anisotropía diamagnética común que presentan estos compuestos. Siendo así, se define un compuesto aromático como " moléculas cíclicas, diatrópicas, moléculas que en el espectro RMN muestran apantallamiento de la corriente anular diamagnética, en la que todos los átomos anulares participan en un único sistema conjugado" ( Garrat, 1974).

Es lógico pensar que la anterior definición no sea la única manera de definir un sistema aromático según algunas propiedades en el estado fundamental ( magnetismo); de hecho se cuenta con las longitudes de enlace entre carbonos, como otro criterio para diferenciar un compuesto aromático de otro que no lo es, aunque en sistemas policiclicos o heterociclicos sea complejo demostrarlo.

Lo anterior sin que se haya modificado en núcleo firme del programa de investigación propuesto anteriormente. 


\section{CONCLUSIONES}

La teoría de la aromaticidad vista como un programa de investigación, se constituye como tal por la solidez de sus postulados, la capacidad para predecir hechos nuevos y vigentes. La consolidación de la teoría impidió que surgieran programas en competencia lo suficientemente fuertes como para sustituir a la propuesta por Kekulé.

El éxito de la teoría de la aromaticidad se debe precisamente a la amplitud constante del núcleo firme, permitiendo dar explicación a las anomalías surgidas durante el desarrollo de la teoría. Aunque Kekulé no propusiera apoyos empíricos a su teoría, contó con los aportes experimentales de otros científicos para darle forma a los postulados que conforman el núcleo firme de este programa.

El programa de investigación propuesto por Kekulé ha logrado hasta hoy dar explicación a un sin número de fenómenos que han surgido producto de las constantes investigaciones sobre los compuestos aromáticos.

La teoría de la aromaticidad, consolida conceptos como valencia, aromaticidad y al mismo tiempo, estos conceptos confirman las explicaciones que sobre la teoría se han planteado. De esta manera, los conceptos que dan cuerpo a teorías como la de los orbitales moleculares, la de Hibridación , propiedades como el magnetismo o las longitudes de enlace, son nuevas ampliaciones al cinturón protector del programa de aromaticidad, sin que el núcleo firme se vea modificado y la teoría por tanto de las explicaciones necesarias de forma satisfactoria.

Como consecuencia del análisis histórico-epistemológico realizado sobre la Teoría de la Aromaticidad propuesta por Kekulé, las autoras de este artículo sugieren que esta teoría, enmarcada bajo la concepción metodológica Lakatosiana, es susceptible de llevarse al aula para ser objeto de estudio mediante una unidad didáctica que busque enseñar los aspectos conceptuales, metodológicos, actitudinales y axiológicos (Gallego Badillo y Pérez Miranda, 1999) ligados al proceso de construcción de la teoría analizada, evitando las tergiversaciones que generalmente se suscitan cuando los procesos de enseñanza no parten de un estudio de los documentos originales.

\section{BIBLIOGRAFÍA}

- ARMSTRONG, H.E.J (1887) Chem. Soc. Pp 51, $258-268$.

- BAEYER, A.(1888) Liebigs Annalen der Chemie, pp 245, 103-185

- BROWN C, (1860) "Models and structural diagrams in the 1860s" Extracts from proceedings of the Royal Institution, 4, pp 401-430

- CLAUS Y KOERNER (1867) "Completion of Koerner's Proof that the Hydrogens Of Benzene are Homotopic. An application of group theory" Journal Amer. Chem. Soc. Pp 102.

- COUPER, A.S.(1858) " On a New Chemical Theory". In: Philosophical Magazine. № 4 Vol. 16. pp 104-116.

- DEWAR, M.J.S. (1945) "Autobiography: Dewar, M J S.A Semiempirical life". Nature. Pp 155, 50-51 
- DEWAR. J, (1866) "Dewar's Brass strip models" From Proceedings of the Royal Society of Edinburg, VI session pp 82-86.

- DUMAS (1840) "On the substitution law and the theory of types (leter to justus Liebig)" Annalen der Chemie and Pharmacie vol 33 pp 308

- FRANKLAND, E. (1852) "On a New series of organic bodies containing metals" Classic in the Theory Of Chemical Combination, New York pp 76-108.

- GAGLIARDI, R. (1988) "Cómo Utilizar La Historia De Las Ciencias En La Enseñanza De Las Ciencias". En: Enseñanza de las ciencias, 6(3), 291-296.

- GAGLIARDI, R. y GIORDAN, A. (1986). "La Historia De Las Ciencias: Una Herramienta Para La Enseñanza". Enseñanza de las ciencias, 4 (3), 253-258.

- GALLEGO, B. Y PÉREZ, M (1997) La enseñanza de las ciencias experimentales. El constructivismo del caos Editorial Magisterio. Bogotá.

- GARRAT, P.J. (1974). Aromaticidad . Madrid, España: ALHAMBRA.

- HOFMAN (1865) "Hoffman's Croquet balls models" Extracts from proceedings of the Royal Institution, 4 pp 401-430.

- KEKULÉ, A.(1865). "Study on aromatic compounds" In: Annalen der Chemie. No 137. pp 129-196.

- LAKATOS, I. (1983) "La metodología de los programas de investigación científica" . Alianza editorial .Madrid, España.

- LANDERBURG (1860). VITORIA . E (1940) "Química del carbono teórica y práctica" 2 Editorial Católica Casals. Barcelona.

- LAVOISIER, A.L. (1789) "Traité élementaire de chemie . Paris (Tratado elemental de Química)". Alfaguara Madrid 1982.

- LOSCHMITH (1860). VITORIA . E (1940) "Química del carbono teórica y práctica" 2 Editorial Católica Casals. Barcelona.

- THIELE, J.(1899) Liebigs Annalen der Chemie pp 306, 87-142

- VAN'T HOFF. J. H, (1874-77) "The arrangement of atoms in space "La Chimie dans l'espace" Francia.

- VITORIA, Eduardo. QUÍMICA DEL CARBONO TEORICA Y PRACTICA. Segunda edición. Editorial Católica.1940. Barcelona, España. pág.179-184.

- WOHLER, F., LIEBIG, J. (1832) "Resaerches respecting the radical of benzoic acid" Classic in the Theory Of Chemical Combination, New York pp 15-39.

- WOODROW, W. (1992). "Friederich August Kekulé: A scientist and Dreamer". In: www.woodrow.org/teachers/chemestry/institut/1992/kekule.html. 